children in a school context; '...E vai con la frutta', good practices to promote and sustain the fresh fruit and vegetables daily consumption in schoolchildren (10-14-16 years); 'Observatory on the lifestyles', with the aim to follow-up the health promotion projects addressed to the contrast of the main risk factors for CVD; 'Ragazzinsieme', in movement between health, environment, culture and traditions (10-17 years).
Results: The overweight prevalence in childhood and adolescence in Tuscany has remained steady for 7 years.

Conclusions: The results can partially be due to the regional strategy that links the single projects in a unique program addressed to the health promotion with the involvement of all sectors.

Funding: Researches relating to this abstract were funded by Tuscany Region, 'Guadagnare Salute in Toscana'.

\title{
74 - PERISCOPE Project: how to translate healthy lifestyle in funny and enjoyable ones to preschool children and their families
}

\author{
M Caroli ${ }^{1}$, E Malecka-Tendera ${ }^{2}$ and BE Mikkelsen ${ }^{3}$ \\ ${ }^{1}$ Nutrition Unit, Department of Prevention, ASL Brindisi, Italy: ${ }^{2}$ Medical University of Silesia, Katowice, Poland: \\ ${ }^{3}$ Aalborg University, Ballerup, Denmark
}

\begin{abstract}
Aim: Aims of PERISCOPE are to increasing the discovery and knowledge of healthy never tasted foods by preschool children, training parents and teachers in eating habits modification techniques, improving children's motor abilities and physical activity levels.

Method: A two-side-book titled: 'Healthy eating the Mediterranean way: Tasty tale for children and practical tips for adults/Exercise the old fashioned way: active games for children' has been written, as health is the result of healthy eating and active lifestyle. The eating style side introduces vegetables, legumes and fruit to children through short tales. In these tales, foods and/or nutrients are positive actors of short stories to reach good health or other positive goals. A chapter with simple food health-related information, eating habits development factors and correct techniques to improve them is dedicated to parents and teachers. The
\end{abstract}

physical activity side consists of a theoretical part on motor abilities development and its relationship to psychosocial development dedicated to parents and teachers. A number of active 'old-street-games' at no cost is listed with all the instructions to perform them. The book has been used in the intervention group, during class meetings with children. Statistical analysis performed by McNemar test.

Results: The children in the intervention group ( $n$ 386) showed an increase of their fruit, vegetables and legumes intake and a higher level of physical activity as compared with the control ones ( $n 301 ; P<0 \cdot 01$ ).

Discussion: A tale book is a positive tool to improve eating habits and physical activity level in preschool children. This abstract arises from the project PERISCOPE, which has received funding from the European Union, in the framework of the Public Health Programme.

doi:10.1017/\$1368980012002492

\section{5 - Associations between stress and obesity in primary-school children}

\author{
N Michels, I Huybrechts, B Vanaelst and S De Henauw \\ 'University of Ghent, Department of Public Health, Ghent, Belgium
}

Introduction: To define correlations between stress and obesity-related factors in first to fourth grade primaryschool children.

Method: A total of 532 children (50\% male) aged 5-11 years were included from the Belgian control region of the EU 6th FP IDE-FICS cohort. BMI, body fat percentage
(BF \%) (using bioelectrical impedance, skin folds and body circumferences) and blood pressure were measured via standardized procedures. To assess stress-inducing lifeevents in the last year, the validated CLES-C questionnaire was completed during personal interview with the child. An age-adjusted score was calculated. Coping style was 